\title{
Outreach, Education and Communication Initiatives of the CTA Observatory
}

\author{
Alba Fernández-Barral ${ }^{a,{ }^{*}}$ and Megan Grunewald ${ }^{a}$ on behalf of the Cherenkov \\ Telescope Array Observatory (CTAO)
}

${ }^{a} \mathrm{CTAO} \mathrm{gGmbH}$,

Saupfercheckweg 1, Heidelberg, Germany

E-mail: alba.fernandezbarralecta-observatory.org

The Cherenkov Telescope Array Observatory (CTAO) will be the first ground-based gamma-ray observatory to explore the extreme Universe, which will be open to all scientific communities as a resource for very high-energy astronomical data. CTAO will have two sites, with two telescope arrays covering the entire gamma-ray sky: one in the northern hemisphere at the Roque de los Muchachos Observatory (La Palma, Spain) and one in the southern hemisphere near the Paranal Observatory (Atacama Desert, Chile). Here, we present some of the Outreach, Education and Communication programmes carried out internationally.

We present the educational material to bring the exotic gamma-ray Universe into the classroom, as well as the "Physicists On-Call" program that put in contact educational centres and astronomical associations with CTA experts around the world. Moreover, we will delve into the Astrodiversity project, whose goal is to create and support initiatives within the inclusion and diversity framework and that includes the annual event "Women of CTA" and a handbook of best practices to make colour-blind friendly material. Finally, we discuss the latest online initiatives that include open seminar series for researchers and general public, and the CTAO series of films to explore the science, technology, sites and people behind the construction of the largest, most powerful gamma-ray observatory on the planet.

\section{*Presenter}




\section{Introduction}

The Cherenkov Telescope Array Observatory (CTAO) will be the first ground-based gamma-ray observatory and the world's largest and most sensitive instrument for the detection of gamma rays. CTAO will transform our understanding of the high-energy Universe by seeking to address a wide range of questions in and beyond astrophysics falling under three major themes: 1. Understanding the origin and role of relativistic cosmic particles, 2. Probing extreme environments, and 3. Exploring frontiers in physics. Moreover, it will be the first of its type to be open to the world-wide astronomical and particle physics communities as a resource for data from unique, high-energy astronomical observations.

CTAO will host dozens of telescopes in two sites: one in the northern hemisphere in the Roque de los Muchachos Observatory on the island of La Palma (Spain), and one in the southern hemisphere near the Paranal Observatory in the Atacama Desert (Chile). The configuration of the telescope arrays for the first construction phase ("Alpha Configuration") includes 4 Large-Sized Telescopes (LSTs) and 9 Medium-Sized Telescopes (MSTs) for the northern hemisphere array, and 14 MSTs and 37 Small-Sized Telescopes (SSTs) for the southern hemisphere array. The CTAO $\mathrm{gGmbH}$ is the interim legal entity for the CTAO in the preparation of the design and implementation of the Observatory until the final legal entity, a European Research Infrastructure Consortium (ERIC), is achieved. The CTAO gGmbH is governed by the CTAO Council, composed of shareholders from 11 countries and one intergovernmental organization, as well as associate members from two countries. CTAO gGmbH works in close cooperation with the CTA Consortium (CTAC) that is contributing to the definition of the instrument design and the scientific programme. CTAC is composed of 1500 members from 25 countries. Thus, CTA is a truly international project, with one-third of its community coming from outside Europe and strong development contributions from the Americas, Africa and Asia.

CTAO's outreach, education and communication strategy has, thus far, been dedicated to the development of a solid foundation and the gradually expansion of initiatives and materials for various groups, including general public, students at all levels, educators, media, local/national communities of the CTA sites and global science community. For the development of these programmes, we always consider the diversity that characterizes the international CTA project. We are very proud of our diversity and aim to be a reference for inclusion and respect in science, as well as to work internationally to achieve equity in our field. Diversity in scientific groups is not only morally fair, but also beneficial for the working environment, increasing creativity and providing different points of view that help to approach and solve problems diversely.

In this document, we report on the various programmes carried out by the CTAO Outreach, Education and Communication team to bring the high-energy gamma-ray astronomy to a broad public within the diversity and inclusion frameworks. It is divided as follows: Section 1 reports on the CTAO educational materials, particularly on the "Physicists On-Call" programme. Section 2 is dedicated to online initiatives, focused on a series of films and webinars. Finally, Section 3 includes some of the activities developed within the CTAO's Astrodiversity project. 


\section{Educational Programmes: "Physicists On-Call"}

One of the main CTAO's outreach and communication targets is the educational community. In order to provide educational material to explore the highest-energy Universe and bring the newly scientific field of gamma-ray astronomy in the classrooms or at home, CTAO has developed a dedicated webpage: "CTA for Educators." This webpage contains tools divided into different educational levels, such as Elementary, High-School, University and "All ages," which helps educators to find the most appropriate material for their students. "CTA for Educators" provides videos and handbooks to work with the youngest, games and worksheets to explore specific topics of the field such as pulsars (Figure 1), explanatory and illustrative posters, as well as the "Dictionary of the Extreme Universe." The latter is a complete list with brief descriptions of typical words used in (very) high-energy astrophysics and astronomy. This dictionary is the perfect tool to help and support different educational activities, including those available at "CTA for Educators." The entire "CTA for Educators" page, and the material therein (including the dictionary), is also available in Spanish in "CTA para Educadores." Tools of both pages are constantly expanding.

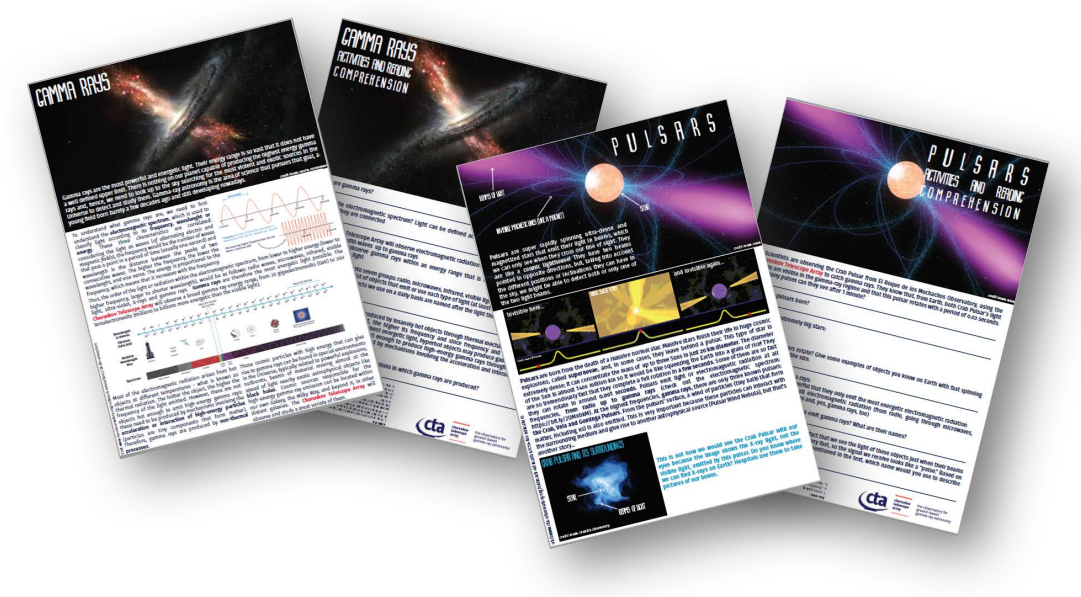

Figure 1. Example of worksheets available at the "CTA for Educators" page for highschool students. Worksheets are composed of two pages: the first one briefly develops a topic of the gamma-ray field, while the second includes reading comprehension activities.

Additionally, CTAO has an educational online programme on-going: "Physicists On-Call." The goal is to bring to the classroom and to astronomy association meetings the very high-energy astronomy, as well as to delve into the innovative CTA technology and its coming scientific achievements by the hand of experts in the field. To do so, any member of an education institution (primary, secondary, collegiate or post-graduate levels) and members of astronomy associations can request a talk from CTA physics or engineering staff through a request form available on the "CTA for Educators" page. While the request form is available in English and Spanish (within "CTA para Educadores"), presentations can be requested in any of the many languages spoken within the CTA project, whose groups are located around the world in 25 countries. 
The programme was released at the end of 2020 and has received 14 requests, reaching 5 countries (Argentina, Italy, Iran, Spain and UK). The panel of CTA speakers that participated in this first year was gender-balanced (four women and four men), and carried out presentations in 5 different languages. After the talks, the contact person for the participating group is asked to fill out a voluntary and anonymous survey to help us improve the programme. "Physicists On-Call" has reached more than 420 people, based on the results of the available surveys, with an average of $\sim 40$ people per talk ${ }^{1}$. The audience we have reached the most has been high-school students of 16-18 years old, although other targets, such as astronomy associations, physics teacher associations and younger high-school students (12-16 years old) are also among the participants. Elementary, university and postgraduate students are targets to reach in future talks. The most discussed topics were general astrophysics and CTA technology (CTA Cherenkov telescopes), following by CTA science (specific science topics, such as dark matter) and the CTA project. While no talk was completely focused on diversity and inclusive topics, such as women in science, the topic was incorporated in the presentations. To the question, "What did the students/audience enjoy the most?", most of the participants said that it was "being able to talk with an expert in the field" (Figure 2), and specifically to talk to and learn firsthand the experience of a woman in astrophysics (added in "Others").

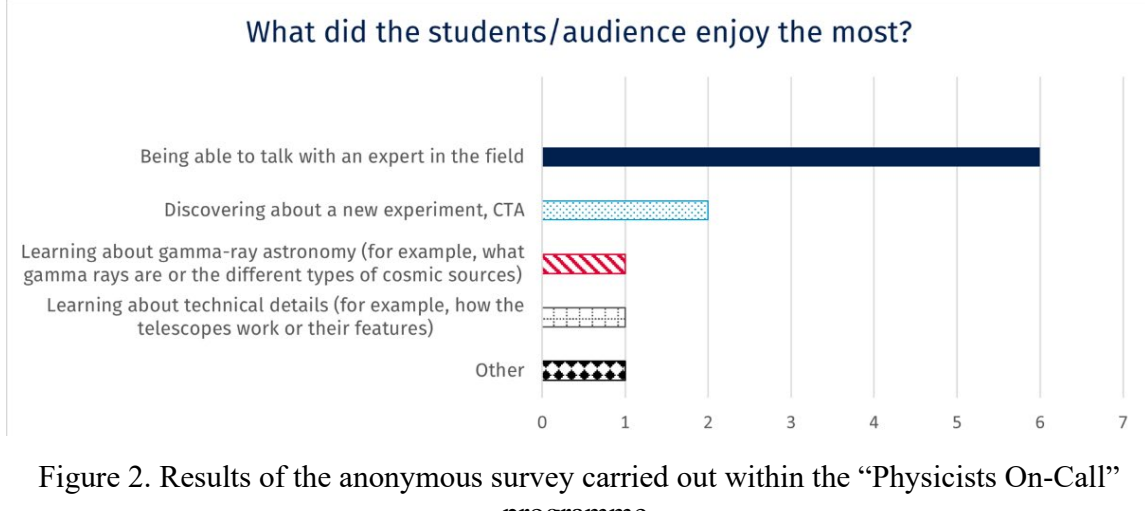
programme.

$90 \%$ of the survey respondents answered that the interest shown by the audience throughout the talk was very high and rated the talk as very good too $(10 \%$ answered high/good, respectively ${ }^{2}$ ). $100 \%$ of the survey respondents considered that it was a "very positive" experience for the students. All of them would recommend the programme and participate again (two of those group have already requested a second talk).

After such positive feedback and great reach (more than 420 participants), "Physicists OnCall" will be carried out again in the next scholar year (2021-2022). The new edition will provide different options to the requesters, as virtual tours to the CTAO sites and chats with CTA experts (without formal presentation). Although initially the programme will remain completely online, when possible, in-person talks will be evaluated.

\footnotetext{
${ }^{1}$ Missing information from some of the talks (waiting for the survey results).

${ }^{2}$ Options: very high/very good, high/good, average/fair, low/poor and very low/very poor.
} 


\section{Online Programmes: Film and Webinar Series}

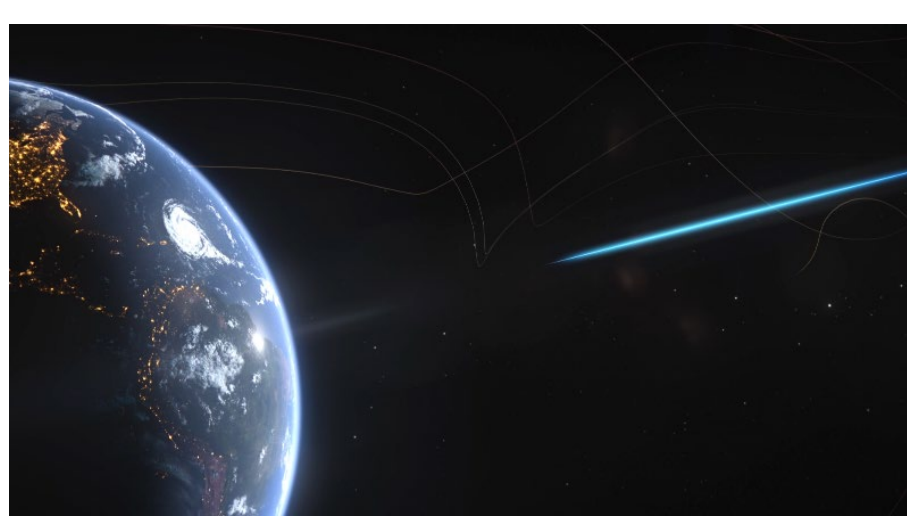

Figure 3. "CTA Science: Emission to Discovery" film. Credit: CTAO $\mathrm{gGmbH}$.

Between 2020-2021, CTAO developed online tools for diverse target groups. One of these tools is a series of films to explore the science, technology, sites and people behind the construction of the largest, most powerful gammaray observatory on the planet. The release of the series initiated in May 2020 with "CTA Science: Emission to Discovery", a science animation that takes the viewer from the emission of gamma rays by extreme sources and the collection of Cherenkov light by CTAO on Earth to data analysis and discovery (Figure 3). The second and the third films explore the northern and southern hemisphere sites, respectively, by the hand of Paolo Calisse (CTAO-North Site Manager) and Volker Heinz (CTAOSouth Site Manager). On 10 June 2020, the film "The CTA-North Site: Our Northern Eye on the High-Energy Universe" was premiered on a live streaming event hosted by Sky-live.tv, which reached almost $\sim 1700$ people on their YouTube channel. The event included presentations about the science and northern hemisphere site, as well as the broadcast of the films. Following the same recipe, on 17 March 2021 and on the occasion of the Astronomy Day in Chile, "The CTA-South Site: Our Southern Eye on the High-Energy Universe" was premiered on a live streaming event co-hosted by the CTAO, European Southern Observatory (ESO) and Pontificia Universidad Católica de Chile (PUC), watched by more than 1,300 viewers on YouTube. In this case, representatives from the CTA Chile community and ESO joined the event to provide a detailed view of the CTA project before broadcasting live the film. The final release of this series, which be launched on the coming months, will cover the full spectrum of the global initiative to build CTA and will include interviews with some of CTA's project team and scientists.

On the other hand, CTAO adapted the outreach and science seminars, typically performed in-person, to the required virtual environment. Thus, in April 2021, two webinar series were launched for different targets.

First, the "Synergies in the Exploration of the Extreme Universe" was launched. This webinar series, organized by the CTAO and CTAC (particularly with the CTA Multi-wavelength Task Force), is focused on the various multi-wavelength (MWL) and multi-messenger (MM) synergies of the CTA science with other instruments and collaborations. Each webinar in the series, which are aimed at researchers in astrophysics and astronomy, take place on the fourth Thursday of every month via Zoom. The time for each webinar alternates to make live connection more accessible to scientists in different time zones. The entire series is open and free to all researchers with a one-time registration through a link on the dedicated webpage. Zoom connection details are sent to subscribers via email a few hours before the event. Additionally, the series programme for each month is advertised on CTA social media (Facebook, Twitter and 
Instagram). The communication is done using the GDPR (General Data Protection Regulation) standards to protect participants data and prevent any spam.

Originally conceived as an instrument to aid CTA MWL Coordination activities, and aimed primarily as a permanent, online discussion forum for the CTA Community, this series was open to all scientists interested in engaging with CTA from the broadest scientific perspectives. The objective is to enhance CTA engagement with the broad astronomy science community, to build bridges with external groups within the MWL and MM fields with which CTA can cooperate in the future, as well as to keep the CTA community up to date about the MWL developments and instrumentation relevant to CTA science. The programme has received great reception, with $\mathbf{4 7 0}$ subscribers as per July 2021.

So far, three webinars have been carried out: Dr. David Thompson (NASA's Goddard Space Flight Center) talked about "Active Galactic Nuclei (AGNs) MWL Research Strategies," Dr. Anna Franckowiak (DESY) delved into the "MM Data Networks," and Dr. Gautham Narayan (University of Illinois at Urbana-Champaign) presented "Alert Brokers for Astrophysical Surveys." A format based on registration to participate in a private Zoom room has proved to be helpful to foster productive discussions among researchers working in the field and consequently synergies and common working projects. Presentations (without the discussion section) and slides are afterwards made available on the $\underline{\mathrm{CTA}}$ website.

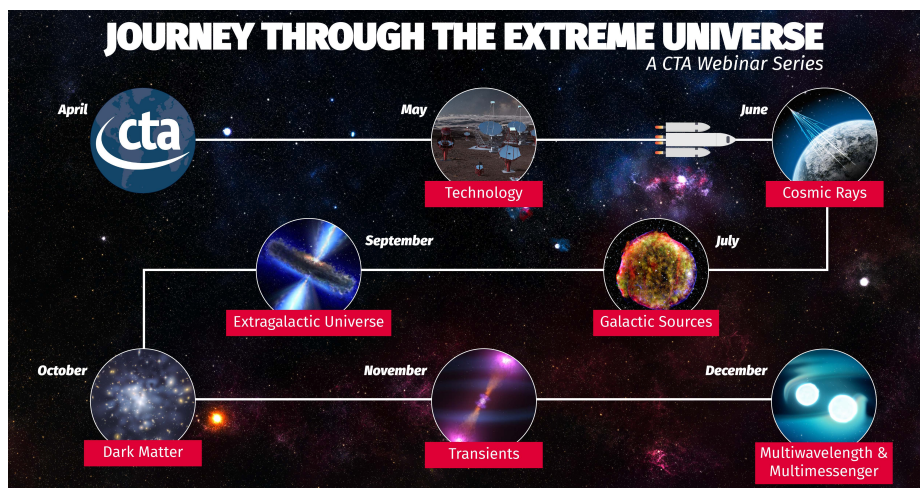

Figure 4. Roadmap of the CTAO's "Journey through the Extreme Universe" webinar series for general public.
The second webinar series launched in April 2021 is the "Journey through the Extreme Universe," aimed at all astronomers fans and general public. This monthly series is livestreamed via the CTA YouTube and Facebook channels. The speakers (or "mission commanders") from the CTAO and CTAC take participants on a journey through the Cosmos to

explore the exciting high-energy Universe in 8 sessions that delve into the CTA Observatory, its technology and CTA science cases (Figure 4). The webinars take place on a Wednesday every month at 17:00 UTC. Recordings are available on a dedicated playlist on YouTube. As per June 2021, the first three webinars have been already performed, with almost 600 views offline in YouTube. After each presentation, a "Question \& Answer" session is carried out, where speakers answer the doubts of attendees. Social media is a good tool for webinars with a more general scope as it is open to everybody and allows interaction between organizers/speakers and participants during the event and afterwards using the comment section.

Both webinar series are regulated by the CTAO Code of Conduct for Events and Meetings. CTAO is committed to supporting and protecting the diversity of the community that brings together in 
each event. This Code of Conduct provides basic standards and rules of behaviour expected in every event organized by CTAO. They apply to any participant, regardless of whether they are CTA members or external participants. It focuses on four core values: Diversity, Integrity, Professionalism and Creativity. For each webinar series, a contact person is defined to whom any violation of the CTAO Code of Conduct for Events and Meetings can be reported. More information and guidelines in each series' webpage.

\section{Inclusive Outreach: Astrodiversity project}

CTA would not be possible without the more than 1,500 members from 25 countries that work together to implement what will be the world's largest and most sensitive gamma-ray detector. In order to foster diversity and inclusion in science, CTAO has created the Astrodiversity program - an initiative to implement and support activities organized under this diversity and inclusion framework. Since its launch in 2019, several Astrodiversity initiatives have already been organized or are being prepared. Here, we report on two: "Women of CTA" and "Best Practices for Colour Blind Friendly Publications and Descriptions."

"Women of CTA" is an annual initiative that takes place on February 11, on the occasion of the International Day of Women and Girls in Science, established by the United Nations in 2015. The pioneering event was initiated in Bologna on 11 February 2019 (in person). Two more editions followed, in 2020 in Bologna still in person and in 2021, the first online edition. "Women of CTA" gathers three CTA experts from the astrophysics and engineering fields to share their academic, professional and personal experience in science and their contributions to CTA (Figure 5). The event was created as part of the global effort to raise awareness and find solutions to gender inequity and the overall under-representation of women in science. Despite international efforts to move equity forward, fundamental for the development of a healthy and productive environment to achieve social and scientific goals, women still represent less than $30 \%$ of the total researchers worldwide [1]. And at an even more fundamental level, girls lose interest in science before starting undergraduate degrees (some of the reasons include gender stereotypes and socialization processes), deterring them from pursuing scientific/technological careers compared to degrees in humanities. As one of the important factors that gives rise to these disparities is the reduced visibility of female leaders and their achievements in STEM (science, technology, engineering and mathematics) careers, projects that foster visibility of female role models in science are fundamental. "Women of CTA" was created to highlight women who are leading and contributing to CTA and to inspire girls and women interested in or already pursuing a STEM career.
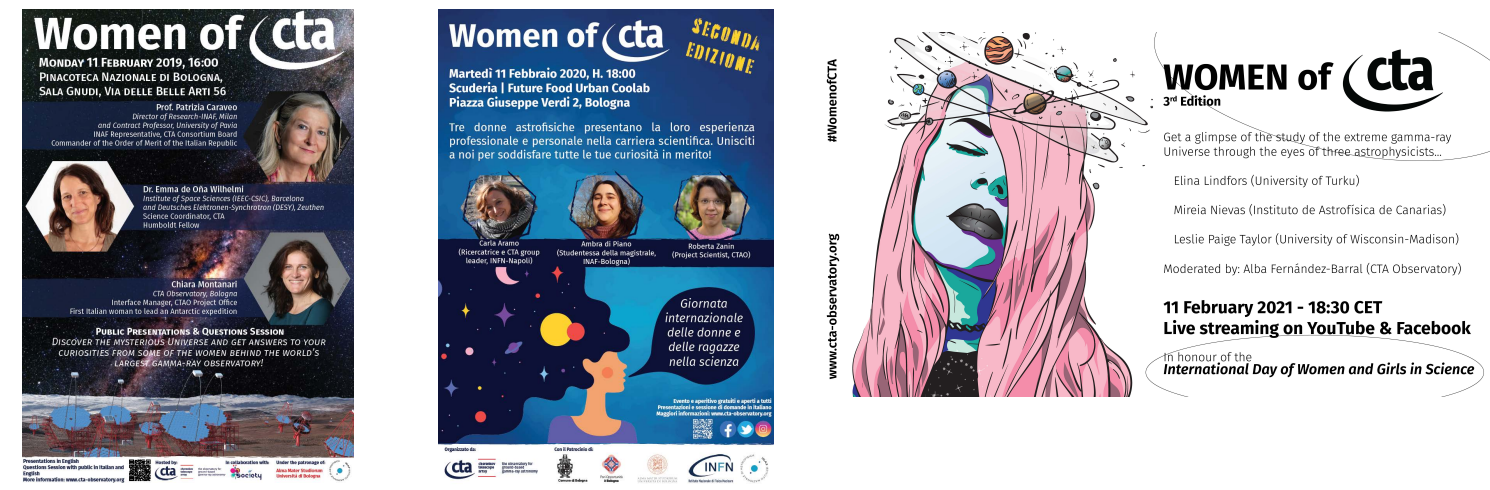

Figure 5. Promotional posters for the three editions of the "Women of CTA" event. 
The first two editions were carried out in-person at Bologna (in English and Italian, respectively) focused on university students. Two locations were used: the Pinacoteca Nazionale di Bologna (Museum at a typical student street in the city) and Scuderia-Future Food Living Lab (a cafeteria with space for students). Both editions had good reception, $\sim 50$ people, especially considering that the February 11 coincides with the exam period for the University of Bologna. The third edition, first online, was livestreamed on the CTA Facebook and YouTube channels in English. The event was viewed by 530 people only on YouTube and got great impact on social media, being the most popular post (the highest engagement) on Twitter and Instagram, and second on Facebook in February. Some "Women of CTA" editions got the support of the University of Bologna, INAF, INFN and Comune di Bologna (with the emblem of "Equal Opportunities at Bologna").

Finally, the CTAO Outreach, Education and Communication team has created the "Best Practices for Colour Blind Friendly Publications \& Descriptions." Colour blindness (or colour vision deficiency, CVD) refers to a reduced capacity or complete incapacity to distinguish colours and affects approximately one in 12 men and one in 200 women [2]. The most common type of CVD is the red-green colour blindness, which despite its name, does not only affect the visibility of red and green but the whole spectrum of colours, as red and green are part of every other colour to some extent.

Considering that CTA is an international and diverse collaboration, whose work reaches the public worldwide, CTAO has created this document, which includes guidelines and tools, including Python packages and usages, to select suitable colours and patterns for different types of plots, as well as to create inclusive descriptions. These guidelines are considered during the review of scientific and outreach material by the CTA Speakers and Publications Office (SAPO) and the CTAO Outreach, Education and Communication team.

The document is available on the CTA website so that anybody can read, share and follow the guidelines to reach a more inclusive work space.

\section{References}

[1] “Cracking the code: girls' and women's education in STEM”, UNESCO, 2017

[2] https://www.color-blindness.com/ 